\title{
3-D RPIC simulations of relativistic jets: Particle acceleration, magnetic field generation, and emission
}

\author{
K.-I. Nishikawa ${ }^{* a}$, P. E. Hardee ${ }^{b}$, C. B. Hededal ${ }^{c}$, Y. Mizuno ${ }^{a}$ and G. J. Fishman ${ }^{d}$ \\ a National Space Science and Technology Center, 320 Sparkman Drive, VP 62, Huntsville, AL \\ 35805, USA \\ E-mail: Ken-Ichi.Nishikawalnsstc.nasa.gov \\ ${ }^{b}$ Department of Physics and Astronomy, The University of Alabama Tuscaloosa, AL 35487, USA \\ ${ }^{c}$ Dark Cosmology Center, Niels Bohr Institute, Juliane Maries Vej 30, \\ 2100 Copenhagen $\emptyset$, Denmark \\ ${ }^{d}$ NASA-Marshall Space Flight Center, National Space Science and Technology Center \\ 320 Sparkman Drive, VP 62, Huntsville, AL 35805, USA
}

\begin{abstract}
Using a 3-D relativistic particle-in-cell (RPIC) code, we have investigated particle acceleration associated with a relativistic electron-positron jet front propagating into an ambient electronpositron plasma without initial magnetic fields in order to investigate the nonlinear stage of the Weibel instability. The growth time and nonlinear saturation levels depend on the initial jet parallel velocity distributions. Simulations show that the Weibel instability created in the collisionless shocks accelerates jet and ambient particles both perpendicular and parallel to the jet propagation direction. The nonlinear fluctuation amplitude of densities, currents, electric, and magnetic fields in the electron-positron shocks are larger for smaller jet Lorentz factor. This comes from the fact that the growth time of the Weibel instability is proportional to the square of the jet Lorentz factor. We have performed simulations with broad Lorentz factor distribution of jet electrons and positrons, which are assumed to be created by the photon annihilation. Simulation results show that the Weibel instability is responsible for generating and amplifying nonuniform, magnetic fields perpendicular to the jet propagation direction and contribute to the electron's (positron's) transverse deflection behind the jet head. This small scale magnetic field structure is appropriate to the generation of "jitter" radiation from deflected electrons (positrons) as opposed to synchrotron radiation. The jitter radiation resulting from small scale magnetic field structures may be important for understanding the complex time structure and spectral evolution observed in gamma-ray bursts or other astrophysical sources containing relativistic jets and relativistic collisionless shocks.
\end{abstract}

VI Microquasar Workshop: Microquasars and Beyond

September 18-22, 2006

Como, Italy

\footnotetext{
*Speaker.
} 


\section{Introduction}

Nonthermal radiation observed from astrophysical systems containing relativistic jets and shocks, e.g., active galactic nuclei (AGNs), gamma-ray bursts (GRBs), and Galactic microquasar systems usually has power-law emission spectra. In most of these systems, the emission is thought to be generated by accelerated electrons through the synchrotron or inverse Compton mechanisms. Radiation from these systems is observed in the radio through the gamma-ray region. Radiation in optical and higher frequencies typically requires particle acceleration in order to counter radiative losses. It has been proposed that the needed particle acceleration occurs in shocks produced by differences in flow speed.

Previous microphysical analyses of the energy conversion in relativistic pair outflows interacting with an interstellar medium consisting of cold protons and electrons have demonstrated that the beam excites both electrostatic and low-frequency magnetohydrodynamic Alfvén-type waves via a two-stream instability in the background plasma [1,2]. These works have also provided the time evolution of the distribution functions of beam particles and the generated plasma wave turbulence power spectra. While in these simulations the jet front showed some evidence of Fermi acceleration, the main acceleration of electrons appeared to take place in the downstream region $[1,2,3]$. Further work in this area is required if significant progress is to be made in unraveling the important collisionless processes in relativistic shocks.

RPIC simulations can shed light on the physical mechanism of particle acceleration that occurs in the complicated dynamics within relativistic shocks. Recent RPIC simulations using injected relativistic electron-ion jets show that acceleration occurs within the downstream jet, rather than by the scattering of particles back and forth across the shock as in Fermi acceleration [4,5,6]. Silva et al. (2003) [7] have presented simulations of the collision of two inter-penetrating electron-positron plasma shells as a model of an astrophysical collisionless shock (see also Jaroschek et al. 2005) [8]. In the electron-positron simulations performed with counter-streaming jets [7,8], shock dynamics involving the propagating jet head (where Fermi acceleration may take place) was investigated by Nishikawa et al. (2005) [9]. In general, these independent simulations have confirmed that relativistic jets excite the Weibel instability [10]. The Weibel instability generates current filaments and associated magnetic fields [11-14] and accelerates electrons [4-9,14,15].

In this paper we present new simulation results of particle acceleration and magnetic field generation for relativistic electron-positron shocks using 3-D RPIC simulations. We present results from three new simulations, in which a cold electron-positron relativistic jet with Lorentz factor, $\gamma V_{\|}=5$ (corresponds to $2.5 \mathrm{MeV}$ ), Lorentz factor $\gamma V_{\|}=15$, and a broad $4<\gamma V_{\|}<100$ Lorentz factor distribution is injected into an electron-positron plasma in order to study the dynamics of a relativistic collisionless shock without an initial ambient magnetic field. The case of a broad Lorentz factor distribution is appropriate to prompt emission at an internal of reverse shock in GRB jets when a pair jet is created by photon annihilation [16].

In the collisionless shock generated, the Weibel instability is excited in the downstream region. The instability generates current filaments elongated along the streaming direction and associated transverse magnetic fields. Acceleration of electrons and positrons in the jet and ambient plasma accompanies the development of the Weibel instability. In §2 the simulation model and initial conditions are described with a broad $4<\gamma V_{\|}<100$ Lorentz factor distribution. The simulation 
results are presented in $\S 3$, and in $\S 4$ we summarize and discuss the new results.

\section{Simulation Setup}

The code used in this study is a modified version of the TRISTAN code, a relativistic particlein-cell (RPIC) code [17]. Three simulations were performed using an $85 \times 85 \times 640$ grid with a total of 380 million particles (27 particles/cell/species for the ambient plasma) and an electron skin depth, $\lambda_{\text {ce }}=c / \omega_{\text {pe }}=9.6 \Delta$, where $\omega_{\text {pe }}=\left(4 \pi e^{2} n_{\mathrm{e}} / m_{\mathrm{e}}\right)^{1 / 2}$ is the electron plasma frequency and $\Delta$ is the grid size. In all simulations jets are injected at $z=25 \Delta$ in the positive $z$ direction. In all simulations radiating boundary conditions were used on the planes at $z=0, z_{\max }$. Periodic boundary conditions were used on all other boundaries [17]. The ambient and jet electron-positron plasma has mass ratio $m_{\mathrm{e}} / m_{\mathrm{p}} \equiv m_{\mathrm{e}^{-}} / m_{\mathrm{e}^{+}}=1$. The electron thermal velocity in the ambient plasma is $v_{\text {th }}=0.1 c$ where $c$ is the speed of light.

In this report we simulate three different initial jet electrons (positrons) distributions. The first case is as same as one of the simulations in a previous paper $[9,15]\left(\gamma V_{\|}=5\right)$, but the system size is 4 or 2 times longer (case A). The second case has a larger Lorentz factor $\left(\gamma V_{\|}=15\right)$ (case B). The third case is a special case and corresponds to cold jet electrons and positrons created by photon annihilation with $4<\gamma V_{\|}<100$ [16]. For all cases the temperature of jet particles are very cold $(0.01 c$ in the rest frame). In this letter we concentrate on the simulation with the broad $4<\gamma V_{\|}<100$ Lorentz factor distribution.

\section{Simulation results}

The electron number density of the jet is $0.741 n_{\mathrm{b}}$ where $n_{b}$ is the ambient electron number density. In this case, the jet makes contact with the ambient plasma at a $2 \mathrm{D}$ interface spanning the computational domain. Here only the dynamics of the propagating jet head and shock region is studied. Effectively we study a small uniform portion of a much larger shock. This simulation system is different from simulations performed using counter-streaming equal number density particles spanning the computational domain in the transverse direction. The important differences between this type of simulation and previous counter-streaming simulations is that the evolution of the Weibel instability is examined in a more realistic spatial way including the motion of the jet head, and we can have different number densities in beam and ambient medium.

Electron density and current filaments resulting from development of the Weibel instability behind the jet front are seen as in other simulations $[5,9,15]$.

The electrons are deflected by the transverse magnetic fields $\left(B_{\mathrm{x}}, B_{\mathrm{y}}\right)$ via the Lorentz force: $-e(\mathbf{v} \times \mathbf{B})$, generated by current filaments $\left(J_{\mathrm{Z}}\right)$, which in turn enhance the transverse magnetic fields $[10,11]$. he complicated filamented structures resulting from the Weibel instability have diameters on the order of the electron skin depth $\left(\lambda_{\text {ce }}=9.6 \Delta\right)$. This is in good agreement with the prediction of $\lambda \approx 2^{1 / 4} c \gamma_{\mathrm{th}}^{1 / 2} / \omega_{\mathrm{pe}} \approx 1.188 \lambda_{\mathrm{ce}}=10 \Delta$ [11]. Here, $\gamma_{\mathrm{th}} \sim 1$ is a thermal Lorentz factor.

The magnetic field energy averaged in the $x-y$ plane is plotted as a function of $z$ (along the jets) at time $t=59.8 / \omega_{\text {pe }}$ in Fig. 1a. The green curves show the perpendicular magnetic field energy $\left(B_{\mathrm{x}}^{2}+B_{\mathrm{y}}^{2}\right)$. The blue curves present the parallel magnetic field energy $\left(B_{\mathrm{z}}^{2}\right)$. Since the parallel magnetic field energy is negligible, the total magnetic field energy (red curves) are overlapped by 
(a)

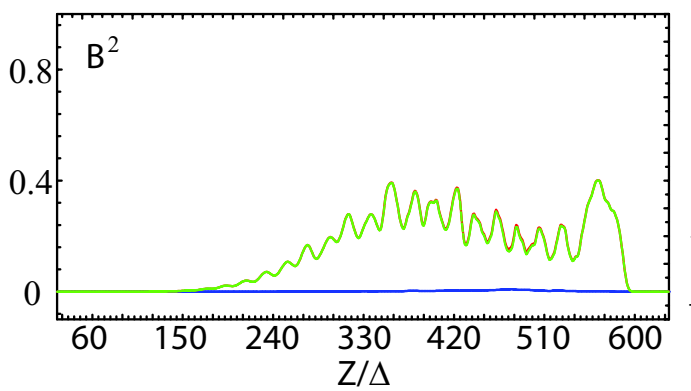

(c)

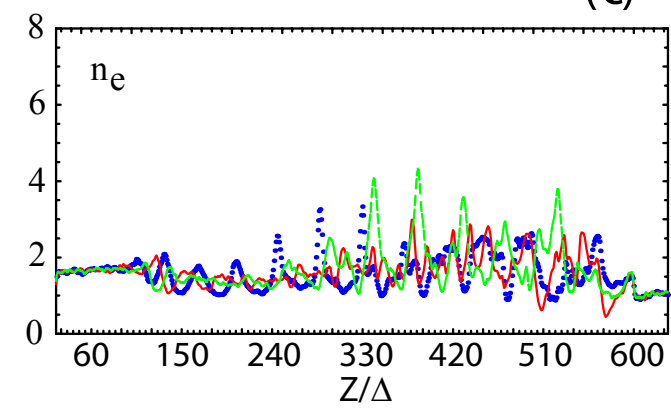

(b)

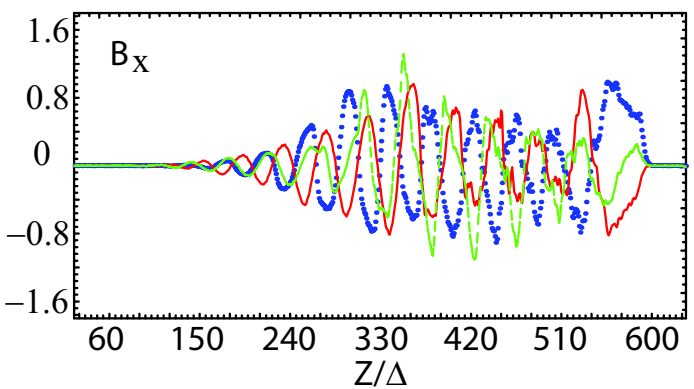

(d)

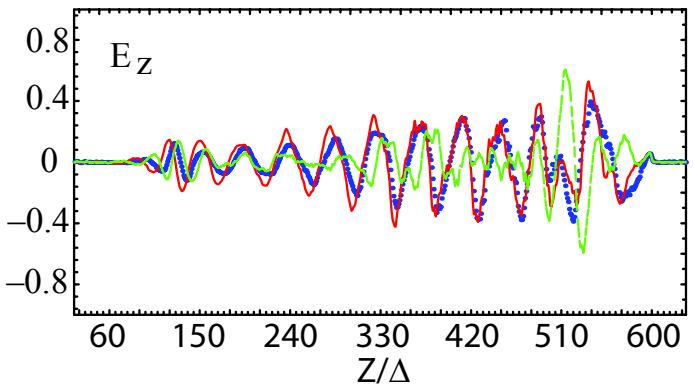

Figure 1: One-dimensional cuts along the $z$-direction of the magnetic field energy density averaged in the $x-y$ plane (a), the $x$-component of the magnetic field (b), the electron density (c), and the $z$-component of the electric field (d) at $t=59.8 / \omega_{\mathrm{pe}}$. The total energy density $\left(B_{\mathrm{x}}^{2}+B_{\mathrm{y}}^{2}+B_{\mathrm{z}}^{2}\right)$ is plotted by the red curves, the perpendicular energy density $\left(B_{\mathrm{x}}^{2}+B_{\mathrm{y}}^{2}\right)$, by green, and the parallel energy density $\left(B_{\mathrm{z}}^{2}\right)$, by blue curves. Since the parallel magnetic field energy (blue) is small, the curves of the total magnetic field energy (red) underlie the perpendicular energy curves (green). The units are simulation units. Cuts are at $x / \Delta=38$ and $y / \Delta=33$ (blue), 43 (red), 53(green), and cuts are separated by about an electron skin depth.

the perpendicular magnetic field energy (green curves). This shows that the Weibel instability generates significant transverse magnetic fields in the jets. Other panels show 1-D cuts through the computational grid parallel to the $z$-axis at $x / \Delta=43$ and $y / \Delta=33,43$, and 53. The spacing in $y$ is about the electron skin depth $\left(\lambda_{\text {ce }} \sim 9.6 \Delta\right)$. This figure provides some quantitative longitudinal information about the filament structures shown qualitatively. With separation by about the electron skin depth, the phase of the instability is different along different 1-D cuts, but the amplitudes are similar. Panel $1 \mathrm{~b}$ shows that $B_{\mathrm{X}}$ decreases for $z / \Delta>350$ and becomes minimum around $z / \Delta=520$. This decrease is smaller than other cases, which is a key issue for this latter. Panel $1 \mathrm{c}$ and $1 \mathrm{~d}$ show the electron density (jet and ambient) and the $z$ component of the electric field, respectively.

Panels 1a and $1 \mathrm{~b}$ show similar structures. Near the jet head a narrow region has large magnetic field energy. Behind that region the magnetic fields become smaller where the current channels created by the Weibel instability merge nonlinearly. The peak at the edge of the linear stage indicates the nonlinear saturation level around $z / \Delta \sim 350$. In case $A$, the saturation level is largest at the linear. However, the magnetic fields dissipate strongly in the nonlinear merging region. In case $\mathrm{B}$ and $\mathrm{C}$ jet particles have higher Lorentz factors, the saturation level is lower and shows less dissipation of magnetic field energy in the nonlinear merging region.

In order to examine the consecutive excitation of the Weibel instability by the broad parallel jet 
(a)

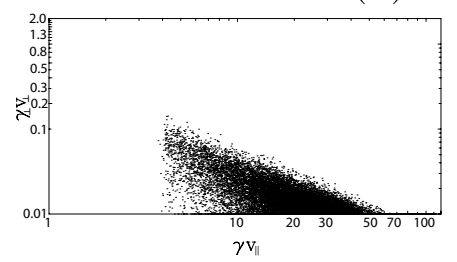

(d)

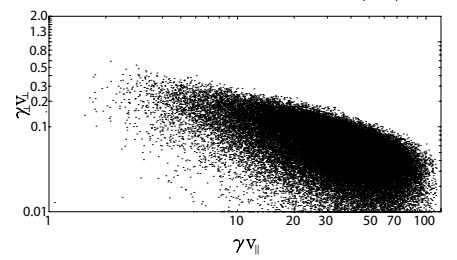

(b)

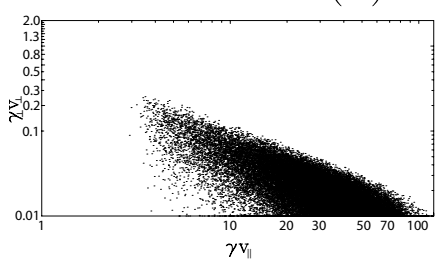

(e)

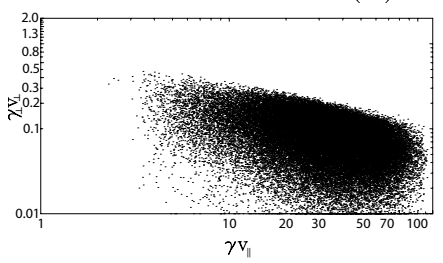

(c)

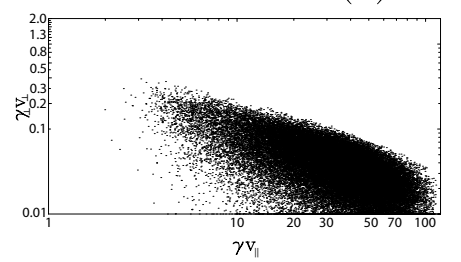

(f)

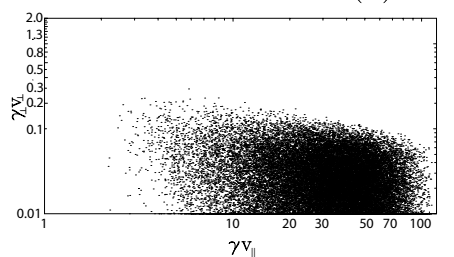

Figure 2: Phase space distributions of jet electrons in $\gamma V_{\|}-\gamma_{\perp} V_{\perp}$ are plotted for at $Z / \Delta=150$ (a), 250 (b), 350 (c), 450 (d), 550 (e), and 588 (f) at $t=59.8 / \omega_{\text {pe }}$. Jet electron are chosen randomly (2.4\%) within $\pm 10 Z / \Delta$ except at $Z / \Delta=588(578<Z / \Delta \leq 600)$.

electron velocity distribution from the lower to higher Lorenz factor the perpendicular acceleration of jet electrons is plotted in Fig. 2. Figure 2 shows the phase space distributions of jet electrons in $\gamma V_{\|}-\gamma_{\perp} V_{\perp}$ are plotted for at $Z / \Delta=150$ (a), 250 (b), 350 (c), 450 (d), 550 (e), and 588 (f) at $t=59.8 / \omega_{\mathrm{pe}}$. In these panels for the perpendicular momentum $\gamma_{\perp} \equiv\left(1-\left(v_{\mathrm{x}}^{2}+v_{\mathrm{y}}\right)^{2} / c^{2}\right)^{-1 / 2}$. Jet electron are chosen randomly (2.4\%) within $\pm 10 Z / \Delta$ except at $Z / \Delta=588(578<Z / \Delta \leq 600)$. Panel 2a shows jet electrons with $\gamma v_{\|}=5$ are accelerated at $140<Z / \Delta<160$, which shows that jet electrons with lower Lorenz factor mainly excite the Weibel instability. This region corresponds the initial linear growth as shown in Fig 1. Panel 2c shows higher perpendicular acceleration with higher Lorentz factor 20 - 30. This suggests that the jet electrons with higher Lorentz factor are also contributing to the excitation of the Weibel instability. The growth time of the Weibel instability is proportional to the Lorentz factor $\gamma_{\mathrm{sh}} / \omega_{\mathrm{pe}}=17.2$ for $\gamma_{\mathrm{sh}}=5$ [11]. The growth time for $\gamma_{\mathrm{sh}}=30$ is around $42 / \omega_{\mathrm{pe}}$, which is within the simulation time $t \omega_{\mathrm{pe}}=58.9$. It should be noted that in the jet front $(570<Z / \Delta \leq 600)$ where the a larger magnetic field exist as shown in F.g 1a and 1b jet electrons are less accelerated in the perpendicular direction as shown in Fig. $2 \mathrm{f}$.

The acceleration of electrons has been reported in previous work [4-9,14,15], which is shown at $t=59.8 / \omega_{\mathrm{pe}}$ in Figure 3. As shown in Fig. 3, particles are accelerated in the perpendicular direction and particles are accelerated and decelerated in the jet direction. The panels in the left and right columns show the jet electron distributions in $Z / \Delta-\gamma V_{\|}$and $Z / \Delta-\gamma V_{\perp}$ phase spaces, respectively. We see that the kinetic energy (parallel velocity) of the jet electrons is transferred to the perpendicular velocity via the electric and magnetic fields generated by the Weibel instability. The strongest transverse acceleration of jet electrons (panel 3b) around $Z / \Delta=560$. The ambient electrons and positrons are also accelerated as shown in Figs. $3 \mathrm{c}$ and $3 \mathrm{~d}$. Some of the ambient electrons are accelerated perpendicularly up to $\gamma V_{\perp}=2$ and are accelerated in the direction of jet flow to greater than $\gamma V_{\|}=2$. The peak of perpendicular acceleration in the ambient electrons occurs around $Z / \Delta=500$. The strongest parallel acceleration in the ambient electrons take place around 
(a)

(b)
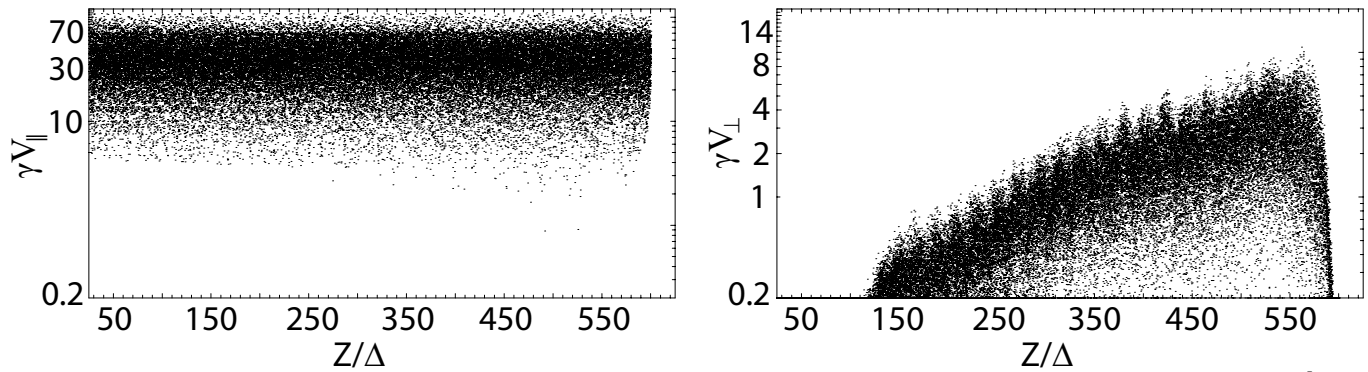

(c)
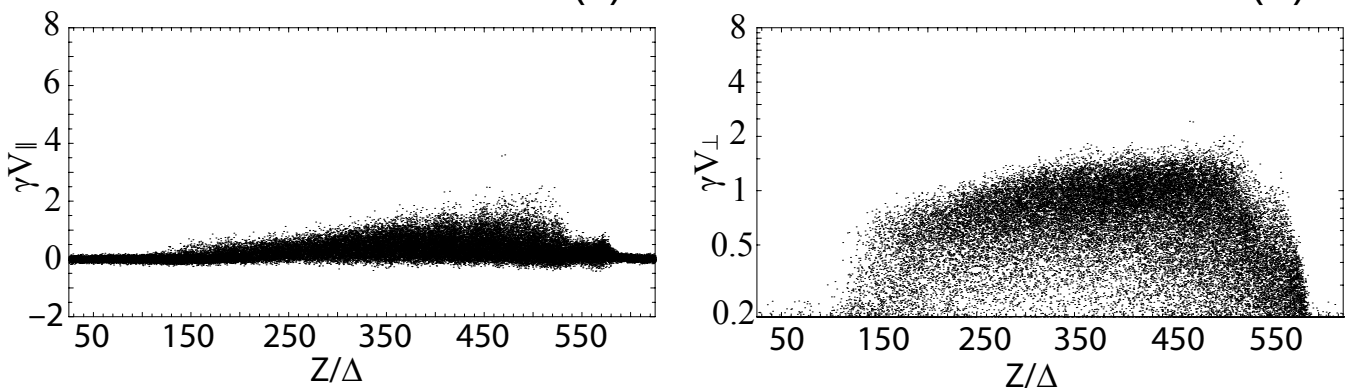

Figure 3: Phase space distributions as a function of $z$ are plotted for the jet electron $(\mathrm{a}, \mathrm{b})$ and the ambient electrons (c, d), in $Z / \Delta-\gamma V_{\|}(\mathrm{a}, \mathrm{c})$ and $Z / \Delta-\gamma v_{\perp}$ phase space $(\mathrm{b}, \mathrm{c})$ at $t=59.8 / \omega_{\mathrm{pe}}$.

the minimum amplitudes of magnetic field energy due to the nonlinear dissipation of the Weibel instability, which is revealed quantitatively in Fig. 1 around $Z / \Delta=500$. Since the electrons and positrons have the same mass, positrons are accelerated equally as electrons.

(a)

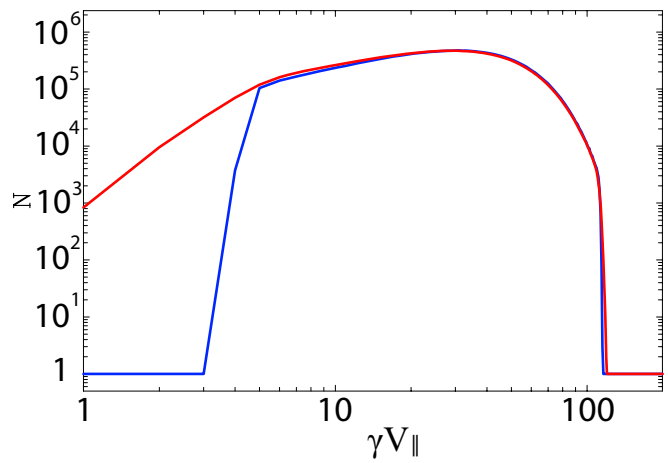

(b)

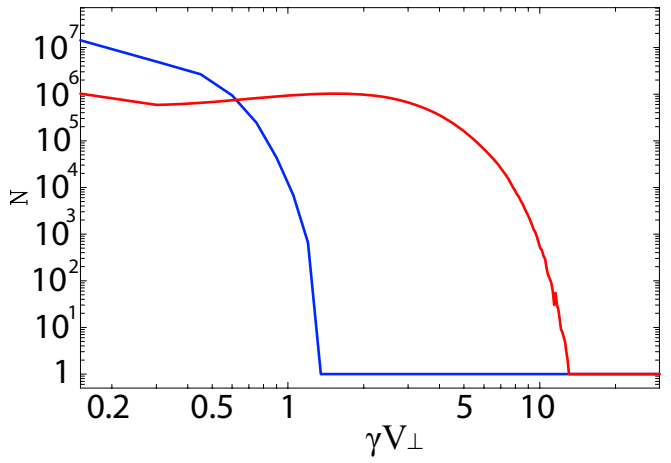

Figure 4: Jet electron parallel (a) and perpendicular (b) momentum distributions are plotted at $t=59.8 / \omega_{\text {pe }}$. The accelerated jet electron distribution is shown by red curves and the initial distribution by blue curves. The zero count on the logarithmic scale is set to 1 .

Figure 4 shows velocity distributions of jet electrons at time $t=59.8 / \omega_{\mathrm{pe}}$. The parallel and perpendicular velocity distributions are shown in panels $4 \mathrm{a}$ and $4 \mathrm{~b}$, respectively. The red curves show the accelerated distributions (found by plotting one third of jet electrons near to the jet front) and the blue curves show the approximately initial distributions (found by plotting one third of 
the jet electrons near to the injection). The average Lorentz factor of this distribution is 36.38 . However, a stronger perpendicular acceleration is found for the higher Lorentz factor and the more broad Lorentz factor distribution (panel $4 \mathrm{~b}$ ). In this case jitter radiation may be more effectively produced as the magnetic fields show less dissipation over a larger region as shown in Fig. 1a and $1 \mathrm{~b}[18-21]$.

\section{Summary and Discussion}

We have performed self-consistent, three-dimensional relativistic particle simulations of relativistic electron-positron jets propagating into unmagnetized electron-positron ambient plasmas for a longer time with a larger simulation system in order to investigate nonlinear stage of the Weibel instability. The Weibel instability originates from the fact that the electrons are deflected by the perturbed (small) transverse magnetic fields accompanying the current filaments and subsequently enhance the current filaments [10-13]. The deflection of particles due to the Lorentz force increases as the magnetic field perturbation grows in amplitude. Our results here are consistent with results from previous simulations $[4-9,14,15]$.

While the jet front showed some evidence of Fermi acceleration, the main acceleration of electrons takes place in the downstream region. Processes in the relativistic collisionless shock are dominated by structures produced by the Weibel instability. This instability is excited in the downstream region behind the jet head, where electron density perturbations lead to the formation of current filaments. The nonuniform electric field and magnetic field structures associated with these current filaments decelerate the jet electrons and positrons, while accelerating the ambient electrons and positrons, and accelerating (heating) the jet and ambient electrons and positrons in the transverse direction. In the nonlinear stage the current channels generated by the Weibel instability merged and are dissipated. The dissipation levels are reduced as the initial jet electron parallel velocity distribution has higher Lorentz factor.

The perturbation amplitudes in the transverse direction become smallest around $Z / \Delta=500$ where nonlinear effects lead to the merging of the smaller scale filaments that first appear behind the jet front. This result is similar to previous counter-streaming simulations $[7,8]$ in which smaller filaments first appear and then merge into larger filaments at a later time.

The efficiencies of conversion of bulk kinetic energy into radiation via synchrotron or "jitter" emission from relativistic shocks will be determined by the magnetic field strength and the electron energy distribution behind the shock. In what follows we examine the conversion of bulk kinetic energy into magnetic and thermal energy by comparing the relevant energy densities in a volume consisting of a number of cells. The simulations show that the initial jet bulk kinetic energy is converted into magnetic energy, transverse acceleration of the jet and ambient particles (thermal energy), and acceleration of the ambient plasma through the Weibel instability.

The generation of magnetic fields both without an initial magnetic field suggests that emission in GRB afterglows and Crab-like pulsar winds could be either synchrotron or jitter emission [18]. The size of filaments is much smaller than can produce observable variations in intensity structure. However, this small size can mean that the deflection angle, $\alpha \sim e B_{\perp} \lambda_{\mathrm{B}} / \gamma m_{e} c^{2}$, of particles by Weibel filaments is smaller than the radiation beaming angle, $\Delta \theta \sim 1 / \gamma$ [18]. Here $\lambda_{\mathrm{B}} \sim \lambda_{\text {ce }}$, $e B_{\perp} / m_{e} c<\Omega_{\mathrm{e}}$, and the ratio $\delta \sim \alpha / \Delta \theta<\Omega_{\mathrm{e}} / \omega_{\mathrm{pe}}$ will be much less than one when the cyclotron 
frequency is less than the plasma frequency. Thus, when ambient magnetic fields are moderate, i.e., the cyclotron frequency is less than the plasma frequency and $\delta \ll 1$, the emission may correspond to jitter rather than synchrotron radiation.

Recent observations show that from optical observations alone the wiggles in the light curves of GRB 011211 are the result of spherically asymmetric density or energy variations, i.e. variations that cover less than the observed $1 / \gamma$ region [22]. The $1 / \gamma$ region has a transverse size of $\sim r / \gamma$ where $r$ is the radial distance to the gamma-ray emitting region. With $10^{14} \leq r \leq 10^{16} \mathrm{~cm}$ the variations need only be somewhat smaller than say $10^{12} \mathrm{~cm}$. Collisionless shocks mediated by the Weibel instability have density and current structures with sizes on the order of the electron skin depth. The typical transverse Weibel filament size $\lambda_{c e} \sim \gamma^{1 / 2} c / \omega_{p e} \sim 3 \times 10^{11} / \omega_{p e}$, where here the relevant value of the plasma frequency in the observer's frame for the Weibel instability is $\omega_{p e} / \gamma^{1 / 2}$. We note that the length of filaments will be subject to length contraction but since the longitudinal plasma frequency is $\omega_{p e} / \gamma^{3 / 2}$ the filament aspect ratio should be preserved. The resultant size for any reasonable estimate of the plasma frequency is many orders of magnitude smaller than the asymmetric density variations implied by the light curves. Thus, the gamma-ray burst may be composed of emission from many different regions but with variation from region to region on much larger scales than those we have considered here. Since we found little difference between no magnetic field and modest magnetic field the Alfvén wave speed was on the order of the thermal speed, we might expect our present results to apply in the presence of magnetic fields with magnetic energy density in equipartition with the thermal energy density.

The injection of $e^{ \pm}$pairs induces strong streaming motions in the ambient medium ahead of the forward shock. As shown in this simulation, the Weibel instability has been excited and magnetic field is generated and sustained for a longer time due to the continuous excitation of the instability by the broad Lorentz factor distribution of the pair electrons [23]. This generated magnetic field in the pair-rich region may provide magnetic field in the afterglow shock [23,24].

The fundamental characteristics of relativistic shocks are essential for a proper understanding of the prompt gamma-ray and afterglow emission in gamma-ray bursts [25], and also to an understanding of the particle reacceleration processes and emission from the shocked regions in relativistic AGN jets $[19,20]$. Since the shock dynamics is complex and subtle, more comprehensive studies are required to better understand the acceleration of electrons, the generation of magnetic fields and the associated emission. This further study will provide the insight into basic relativistic collisionless shock characteristics needed to provide a firm physical basis for modeling the emission from shocks in relativistic flows.

\section{Acknowledgments}

K.N. acknowledges partial support by National Science Foundation awards ATM-0100997, INT-9981508, and AST-0506719, and the National Aeronautic and Space Administration award NNG05GK73G to the University of Alabama in Huntsville. P. H. acknowledges partial support by National Space Science and Technology Center (NSSTC/NASA) cooperative agreement NCC8256 and NSF award AST-0506666 to the University of Alabama. Y. M. is a NASA Postdoctoral Program fellow at NASA Marshall Space Flight Center. The simulations have been performed on 
the IBM p690 at the National Center for Supercomputing Applications (NCSA) which is supported by the NSF.

\section{References}

[1] J. J. Brainerd, A plasma instability of gamma-ray burst emission, ApJ 538 (628) 2000

[2] R. Schlickeiser, R. Vainio, M. Böttcher, I. Lerche, M. Pohl, and C. Schuster, Conversion of relativistic pair energy into radiation in the jets of active galactic nuclei, A\&A 393 (69) 2002.

[3] M. Ostrowski, and J. Bednarz, J. Comment on the first-order Fermi acceleration at ultra-relativistic shocks, A\&A 394 (1141) 2002.

[4] J. T. Frederiksen, C. B. Hededal, T. Haugbølle, and Å. Nordlund, Magnetic field generation in collisionless shocks: Patern growth and trnasport, ApJ 608 (L13) 2004.

[5] K.-I. Nishikawa, P. Hardee, G. Richardson, R. Preece, H. Sol, and G. J. Fishman, Particle acceleration in relativistic jets due to Weibel instability, ApJ 595 (555) 2003.

[6] C. B. Hededal, T. Haugbølle, J. T. Frederiksen, and Å. Nordlund, Non-Fermi Power law Acceleration in Astrophysical Plasma Shocks, ApJ 617 (L107) 2004.

[7] L. O. Silva, R. A. Fonseca, J,.W. Tonge, J. M. Dawson, W.B. Mori, and M. V. Medvedev, Interpretating plasma shells: Near-equipartition magnetic field generation and nonthermal particle accelration, ApJ 596 L121 2003.

[8] C. H. Jaroschek, H. Lesch, and R. A. Treumann, Ultrarelativistic plasma shell collisions in gamma-ray burst sources: Dimensional effects on the final steady state magnetic field, ApJ 618 (822) 2005.

[9] K.-I. Nishikawa, P. Hardee, G. Richardson, R. Preece, H. Sol, and G. J. Fishman, Particle accelration and magnetic field generation in electron-positron relativistic shocks, ApJ 622 (927) 2005.

[10] E. S. Weibel, Spontaneously Growing Transverse Waves in a Plasma Due to an Anisotropic Velocity Distribution, Phys. Rev. Lett. 2 (83) 1959.

[11] M. V. Medvedev, and A. Loeb, Generation of magnetic field in the relativistic shock of gamma-ray burst sources, ApJ 526 (697) 1999.

[12] J. Pruet, K. Abazajian, and G. M. Fuller, New connection between central engine weak physics and the dynamics of gamma-ray burst fireballs, Phys. Rev. D. 64 (063002-1) 2001.

[13] A. Gruzinov, Gamma-ray burst phenomenology, shock dynamo, and the first magnetic fields, ApJ $\mathbf{5 6 3}$ (L15) 2001.

[14] C. B. Hededal, K.-I. Nishikawa, The Influence of an Ambient Magnetic Field on Relativistic collisionless Plasma Shocks, ApJ 623 (L89) 2005.

[15] K.-I. Nishikawa, P. Hardee, C. B. Hededal, and G. J. Fishman, Acceleration mechanics in relativistic shocks by the Weibel instability, ApJ 642 (1267) 2006.

[16] P. Meszaros, E. Ramirez-Ruiz, and M. J. Rees, $e^{ \pm}$pair cascades and precursors in gamma-ray bursts, ApJ 554 (660) 2001.

[17] O. Buneman, Tristan, in Computer Space Plasma Physics: Simulation Techniques and Software, ed. by H. Matsumoto Matsumoto \& Y. Omura, p. 67, Terra Scientific Publishing Company, Tokyo, 1993. 
[18] M.V. Medvedev, Theory of "jitter" radiation from small-scale random magnetic fields and prompt emission from gamma-ray burst shocks, ApJ 540 (704) 2000.

[19] M.V. Medvedev, The therory of spectral evolution of the gamma-ray burst prompt emission, ApJ 637 (869) 2006.

[20] G. D. Fleishman, Diffusive synchrotron radiation from relativistic shocks, ApJ 638 (348) 2006.

[21] C. B. Hededal, Gamma-ray burst, collisionless shocks and synthetic spectra, PhD thesis, 2005. [astro-ph/0506559]

[22] P. Jakobsson, J. Hjorth, E. Ramirez-Ruiz, E. 12 more, Small-scale variations in the radiating surface of the GRB 011211 jet, New Astron, 9 (435) 2004.

[23] E. Ramirez-Ruiz, K.-I. Nishikawa and C. B. Hededal, $e^{ \pm}$pair loading and the origin of the upstream magnetic field in GRB schocks, ApJL, in preparation, 2006.

[24] Z. Li, and E. Waxman, The upstream magnetic field of collisionless GRB shocks, ApJ, in press, 2006. astro-ph/0603427

[25] P. Mészáros, Gamma-ray bursts, Rep. Prog. Phys. 69 (2259) 2006 [astro-ph/ 0605208 ]. 